\title{
Surface structure and stability of PdZn and PtZn alloys: Density-functional slab model studies
}

\author{
Zhao-Xu Chen, Konstantin M. Neyman, Aleksey B. Gordienko, and Notker Rösch* \\ Institut für Physikalische und Theoretische Chemie, Technische Universität München, 85747 Garching, Germany
}

(Received 5 December 2002; revised manuscript received 7 March 2003; published 25 August 2003)

\begin{abstract}
Geometric parameters of binary (1:1) PdZn and PtZn alloys with $\mathrm{CuAu}-L 1_{0}$ structure were calculated with a density functional method. Based on the total energies, the alloys are predicted to feature equal formation energies. Calculated surface energies of PdZn and PtZn alloys show that (111) and (100) surfaces exposing stoichiometric layers are more stable than (001) and (110) surfaces comprising alternating $\mathrm{Pd}(\mathrm{Pt})$ and $\mathrm{Zn}$ layers. The surface energy values of alloys lie between the surface energies of the individual components, but they differ from their composition weighted averages. Compared with the pure metals, the valence $d$-band widths and the Pd or Pt partial densities of states at the Fermi level are dramatically reduced in PdZn and PtZn alloys. The local valence $d$-band density of states of $\mathrm{Pd}$ and $\mathrm{Pt}$ in the alloys resemble that of metallic $\mathrm{Cu}$, suggesting that a similar catalytic performance of these systems can be related to this similarity in the local electronic structures.
\end{abstract}

DOI: 10.1103/PhysRevB.68.075417

PACS number(s): 68.03.Cd, 82.45.Jn, 71.20.Be

\section{INTRODUCTION}

Searching for new sources of hydrogen as an alternative energy carrier has become an important problem because, unlike more harmful conventional fuels, $\mathrm{H}_{2}$ can be used efficiently with negligible emission of greenhouse gases and air pollutants. ${ }^{1}$ One of the ways to produce hydrogen is catalytic steam reforming of methanol. For this reaction, $\mathrm{Pd} / \mathrm{ZnO}$ catalysts have been suggested to replace the currently employed supported $\mathrm{Cu}$ catalysts that sinter at elevated temperature. ${ }^{2}$ The $\mathrm{Pd} / \mathrm{ZnO}$ and $\mathrm{Cu}$ catalysts perform similarly to form $\mathrm{H}_{2}$ and $\mathrm{CO}_{2}$ from methanol, but their catalytic activity differs notably from that of pure metallic Pd. ${ }^{3,4}$ The high performance of $\mathrm{Pd} / \mathrm{ZnO}$ catalysts was assigned to $\mathrm{PdZn}$ alloys recently identified as $\mathrm{PdZn}(1: 1 \mathrm{Pd}: \mathrm{Zn}$ atomic ratio) and $\mathrm{Pd}_{3.9} \mathrm{Zn}_{6.1},{ }^{5,6}$ catalytic properties of alloys formed by $\mathrm{Pt}$ and $\mathrm{Zn}$ are similar. ${ }^{7}$

The electronic and geometrical structure of palladium and platinum alloys, mainly with transition metals in $d^{10-x}$ configurations as second component, was intensively studied due to the industrial importance of $\mathrm{Pd}$ and $\mathrm{Pt}^{8,9}$ At variance, PdZn and PtZn alloys attracted notably less attention. ${ }^{10,11}$ For PdZn alloys, photoelectron spectroscopy showed that alloying studied for PdZn films increases the binding energy of Pd levels. ${ }^{10}$ No information about the relative stability of various surfaces of PdZn and PtZn alloys is available, but such data are necessary for predicting favorable structural arrangements exhibited by surface atoms of these materials. In the absence of even basic knowledge about the surface structure of PdZn and PtZn alloys, it is rather difficult to elucidate the reactivity of these prospective catalysts at the microscopic level. Indeed, it is impossible to study the reaction mechanism on a solid catalyst at the atomic level without having identified the surface sites of the material under investigation. This knowledge relates directly to the issue of relative surface stability which in turn can be rationalized based on information about the surface energy. The latter is a property that determines the equilibrium shape of mesoscopic crystals and plays an important role in faceting and roughing. Direct measurement of surface energies is difficult, especially for such complex solids as alloys. In this respect, one increasingly relies on computational approaches that become more and more powerful. During the past decade surface energies of metals ${ }^{12-14}$ and various alloys were calculated, ${ }^{15,16}$ but no data for PdZn and PtZn alloys are available.

We carried out a comparative computational study on five systems: PdZn and PtZn (1:1) alloys as well as $\mathrm{Cu}, \mathrm{Pd}$, and Pt metals, which all are relevant to the catalytic steam reforming of methanol. We applied a density functional (DF) method in combination with a slab model approach to explore the energetics, geometric and electronic structures of the alloys. Analyzing in parallel the surface (and bulk) properties of the well-characterized $\mathrm{Cu}$ and $\mathrm{Pd}$ reference systems allows one to better trace the most important similarities and differences in the electronic and structural features of the materials under investigation. In the next section we outline the computational details and the procedure of surface energy calculations. The optimized bulk structural parameters of PdZn and PtZn alloys are considered in Sec. III A. The surface energies of various PdZn and PtZn surfaces are addressed in Sec. III B. Section. III C is devoted to surface structures of $\mathrm{PdZn}$. The electronic structure features are discussed in Sec. III D. Our conclusions are presented in Sec. IV.

\section{METHODOLOGY}

\section{A. Computational details}

The calculations were performed with the plane-wave based Vienna ab initio simulation package (VASP) ${ }^{17-21} \mathrm{em}$ ploying the PW91 generalized gradient approximation (GGA). ${ }^{22}$ The choice of the GGA, despite an overestimation of the lattice constants and underestimation of the surface energies at this level (see below), is justified by our primary interest in the relative stability of various surfaces and, more importantly, by the need of our future calculations of the energetics of various chemisorption complexes on the metals and alloys considered here. The interaction between atomic cores and electrons treated explicitly is described by the pro- 
jector augmentation wave (PAW) method using $s, p$, and $d$ waves inside all atomic spheres. ${ }^{23,24}$ Standard values were used as sphere radii to define the PAW space partitioning. ${ }^{24}$ Brillouin-zone integration was done with Monkhorst-Pack grids ${ }^{25}$ using a generalized Gaussian smearing method. ${ }^{26}$ Experimental atomic positions of Pd (fcc), Pt (fcc), Zn (hcp), $\mathrm{Cu}$ (fcc), PdZn (CuAu L1 $1_{0}$ ), and PtZn $\left(\mathrm{CuAu} L 1_{0}\right)$ were taken as starting parameters for the geometry optimization. PdZn and PtZn alloys exhibit a tetragonal CuAu $L 1_{0}$ structure which is derived from an fcc structure by shortening the $c$ axis. $^{27}$

In the slab model calculations, a $400 \mathrm{eV}$ energy cutoff (the same as for bulk) and a $(7 \times 7 \times 1) k$-point grid were adopted. Unit cells containing two atoms per layer were used throughout. Each atomic layer parallel to the (111) and (100) planes of $L 1_{0}$-type $M Z n(M=\mathrm{Pd}, \mathrm{Pt})$ alloys is stoichiometric. On the other hand, each of the (001) and (110) planes contains either $M$ or $\mathrm{Zn}$ atoms, in alternating fashion. We refer to two neighboring (001) and (110) atomic planes of $M Z n$ alloys as one layer in our slab calculations; thus, an $N$-layer slab model comprises $2 N(110)$ and (001) planes and $N$ (111) and (100) layers. The effect of surface relaxation was examined by optimizing the top two layers on one side of $\operatorname{PdZn}(111)$ slabs with four and seven layers, separated by a seven-layer vacuum spacing. The results were very similar. For example, both models predict equal vertical displacements $(-0.11 \AA)$ of Pd atoms in the top layer and an energy gain of $33 \mathrm{meV}$. We also relaxed the first three layers of a five-layer slab and found no significant difference from the results of two-layer relaxation of a four-layer slab. Therefore, all relaxation results discussed below correspond to fourlayer slabs with the top two layers relaxed.

Densities of states (DOS) were calculated with $(16 \times 16$ $\times 16)$ and $(15 \times 15 \times 1)$ k-point grids for bulk and sevenlayer unrelaxed slabs, respectively. We employed the tetrahedron method with Blöchl corrections. ${ }^{28}$ Local and partial DOS were obtained by projecting the wave functions inside a sphere around each ion onto spherical harmonics. For PdZn and PtZn, we chose the sphere radii of Pd, Pt, and $\mathrm{Zn}$ by scaling the corresponding covalent radii ${ }^{29}$ such that the sum of the corresponding sphere volumes equals the volume of the underlying unit cell. The resulting radii are $1.535 \AA$ for Pd in PdZn, $1.545 \AA$ for Pt in PtZn, $1.494 \AA$ for $\mathrm{Zn}$ in both alloys, and $1.418 \AA$ for $\mathrm{Cu}$. In the pure metals $\mathrm{Pd}$ and $\mathrm{Pt}$, spheres with the above radii formally account for about $98 \%$ of the theoretical bulk volume; complete space filling would require to increase the radii to $1.545 \AA$ (Pd) and $1.558 \AA(\mathrm{Pt})$. The results obtained with the two sets of radii were very similar; for example, the center of the $d$-band ( $d$-BC) of bulk Pd changed only by $0.01 \mathrm{eV}$. Sphere overlap was found to have rather minor effects on features of DOS. For consistency, we used the first set of radii (resulting in $\sim 98 \%$ filling) in DOS calculations of Pd and Pt as well.

\section{B. Calculation of surface energies}

The surface energy $\gamma$ is the surface excess free energy per unit area of a particular crystal facet. A common method for calculating surface energies is based on the total energy difference of an $N$-layer slab $E_{S}^{N}$ and of the corresponding bulk $E_{B}$ (Ref. 30)

$$
\gamma=\left(E_{S}^{N}-N E_{B}\right) /(2 A),
$$

where $A$ is the surface area of the unit cell. The factor of $1 / 2$ accounts for the two surfaces of the slab model. In principle, when $N$ is sufficiently large, the calculated value of $\gamma$ will converge to the "true" surface energy. To use Eq. (1), two calculations are required: one for $E_{S}^{N}$ at a reasonably large value of $N$ and the other for $E_{B}$ on the bulk. However, with this method, care has to be taken to ensure consistency between slab and bulk calculations to prevent divergence of $\gamma$ for large values of $N .^{30}$

Surface energies can be determined in reliable fashion by performing a series of calculations on slabs of increased thickness. Then, one derives the surface energy and the bulk energy of a system from a linear fit of the calculated total energy values $E_{S}^{N}$ with respect to the thickness $N$ of the slab: ${ }^{30,31}$

$$
E_{S}^{N}=2 A \gamma+N E_{B} .
$$

This fast converging procedure has been used successfully, ${ }^{13,32}$ but one has to determine the minimum number $N$ of layers from which on the energy $E_{S}^{N}$ exhibits a linear behavior in the slab thickness. ${ }^{32}$ In the systems under investigation, the dependence is essentially linear already for very thin slabs. For example, the $\operatorname{PdZn}(111)$ surface energy derived from fitting of $E_{S}^{N}$ in various ranges, $N=1-7,2-7$, $3-7$, and $4-7$, are $1.11,1.14,1.17$, and $1.18 \mathrm{~J} \mathrm{~m}^{-2}$, respectively, with standard deviations less than $0.04 \mathrm{~J} \mathrm{~m}^{-2}$ and correlation coefficients larger than 0.9999 . These results manifest a nicely linear behavior of $E_{S}^{N}$ on $N$. The surface energies given below correspond to energy fits for the range $N=3$ -7. Note that for (001) and (110) surfaces of PdZn and $\mathrm{PtZn}$, the computed surface energy is actually an average for surfaces terminated by $\mathrm{Pd}$ (or Pt) and $\mathrm{Zn}$ atoms.

First-principles studies of metal surfaces ${ }^{33-35}$ showed that surface relaxation causes variations of $2-5 \%$ in the calculated surface energies. Our calculations for PdZn(111) (see Sec. III C) reveal that the difference in surface energy due to relaxation is $4 \%$. As the purpose of this work is to examine the relative stability of various surfaces, all surface energies presented in the following, unless explicitly indicated, were calculated without accounting for surface relaxation.

\section{RESULTS AND DISCUSSIONS}

\section{A. Bulk structure of PdZn and PtZn alloys}

Metallic $\mathrm{Cu}, \mathrm{Pd}$, and Pt exhibit an fcc crystal structure with lattice parameters of $3.61,3.89$, and $3.92 \AA$, respectively. ${ }^{36} \mathrm{Zn}$ has an hcp structure with the lattice constants $a=2.6649 \AA$ and $c=4.9468 \AA .{ }^{36} \mathrm{Pd}$ and $\mathrm{Zn}$ as well as $\mathrm{Pt}$ and $\mathrm{Zn}$ are very miscible and form alloys in a large range of compositions, ${ }^{27}$ different compositions have different structures. An alloy with an atomic ratio of $\mathrm{Pd}$ to $\mathrm{Zn}$ close to 1 has the structure of $\mathrm{CuAu}$ ( $L 1_{0}$-type) with space group 
P4/mmm (Fig. 1). ${ }^{27}$ In PdZn, Pd, and Zn layers alternate in (001) and (110) directions whereas each crystal plane of (100) and (111) orientations is stoichiometric. The experimental lattice parameters of PdZn alloys at 44.5 at. \% $\mathrm{Zn}$ and 55.2 at. $\% \mathrm{Zn}$ are $a=4.13 \AA$, $c / a=0.81$, and $a=4.09 \AA$, $c / a=0.82$, respectively. ${ }^{27}$ The interpolated values for 50 at. $\% \mathrm{Zn}$ are $a=4.11 \AA$ and $c / a=0.815$. PtZn alloy with an atomic ratio close to $1: 1$ also features a $\mathrm{CuAu}\left(L 1_{0}\right.$ type) structure; the lattice parameters at 47.2 at. $\% \mathrm{Zn}$ are $a$ $=4.03 \AA$ and $c / a=0.860 .{ }^{27}$

Optimized bulk geometries of 1:1 PdZn and PtZn alloys as well as for pure metals $\mathrm{Cu}, \mathrm{Pd}, \mathrm{Pt}$, and $\mathrm{Zn}$ are shown in Table I. It is well known that GGA overestimates the interatomic distances, in particular for $4 d$ and $5 d$ transition metals, in line with our calculated lattice parameters for Pd and $\mathrm{Pt}$; agreement is better for $\mathrm{Cu}$. For the alloys PdZn and PtZn the computed values of $a$ and $c$ are also slightly larger than the experimental estimates, resulting in the $c / a$ ratio very close to experimental value.

In Table II we collected calculated interatomic and interlayer distances for four crystal planes of the two alloys and we compared them to interlayer distances of Pd and Pt metals. The nearest-neighbor distance is $\left(a^{2}+c^{2}\right)^{1 / 2} / 2$ and the next-nearest-neighbor distance is $a / 2^{1 / 2}$. In the fcc structure of $\mathrm{Pd}(\mathrm{Pt})$, the calculated lattice parameters are $a=c$ $=3.95 \AA(3.99 \AA)$; for the tetragonal structure of PdZn $(\mathrm{PtZn})$, the results are $a=4.15 \AA(4.09 \AA), c=3.39 \AA(3.52$ $\AA$ ); the $c$ values of PdZn and PtZn are much shorter than the lattice constants $a$ which, on the other hand, are only slightly longer than the lattice constant $a$ of the corresponding metal $\mathrm{Pd}$ or Pt. As a consequence, each atom of the 1:1 alloys has eight nearest-neighbor heteronuclear bonds and four nextnearest-neighbor homonuclear bonds. Note that nearestneighbor and next-nearest neighbor distances of PdZn differ by $0.26 \AA$ while this difference for PtZn is notably smaller, $0.19 \AA$.

The interlayer distances $d_{\text {int }}$ between neighboring crystal planes in the alloys are as follows: $c / 2(001), 2^{1 / 2} a / 4$ (110), $a / 2$ (100), and $a c /\left(a^{2}+2 c^{2}\right)^{1 / 2}$ (111). These expressions help to understand the variation of the calculated values $d_{\text {int }}$ in Table II. For example, as $c<a$ in the tetragonal structure of PdZn and PtZn, $d_{\text {int }}^{100}$ is larger than $d_{\text {int }}^{001}$ whereas in the fcc structures of Pd and Pt they are equal. Compared to Pd and Pt metals, $d_{\text {int }}^{001}$ and $d_{\text {int }}^{111}$ values in the alloys are reduced whereas $d_{\text {int }}^{100}$ and $d_{\text {int }}^{110}$ values are increased.

In Table I we also list the calculated bulk (cohesive) energies $E_{B}$ with respect to the spin-polarized ground state energies of the constituting atoms. Interestingly, alloy formation from bulk metals $M$ and $\mathrm{Zn}$ is characterized by equal energy values per pair $M Z \mathrm{Zn}, \Delta E=2 E_{\mathrm{MZn}}-\left(E_{M}+E_{\mathrm{Zn}}\right)$ $=-1.15 \mathrm{eV}$. Here, negative values indicate that alloy formation is energetically favorable, in agreement with the large miscibility of $\mathrm{Pd}$ and $\mathrm{Pt}$ with $\mathrm{Zn}$.

\section{B. Surface geometry of PdZn alloy}

Calculated structural parameters of various surfaces of the alloy PdZn are collected in Table III. On the stoichiometric (111) and (100) surfaces, Pd atoms of the first layer $\left(\mathrm{Pd}^{1}\right)$

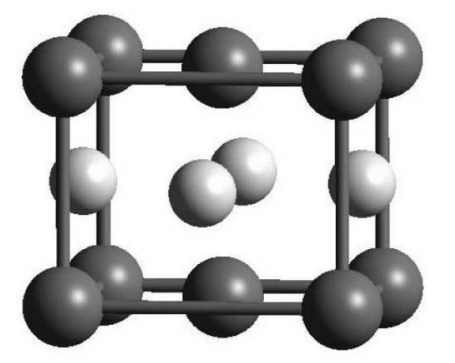

a

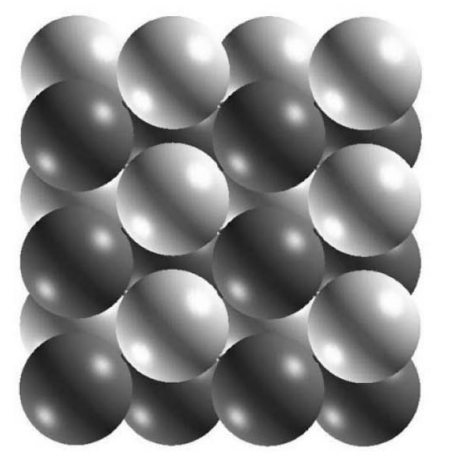

b
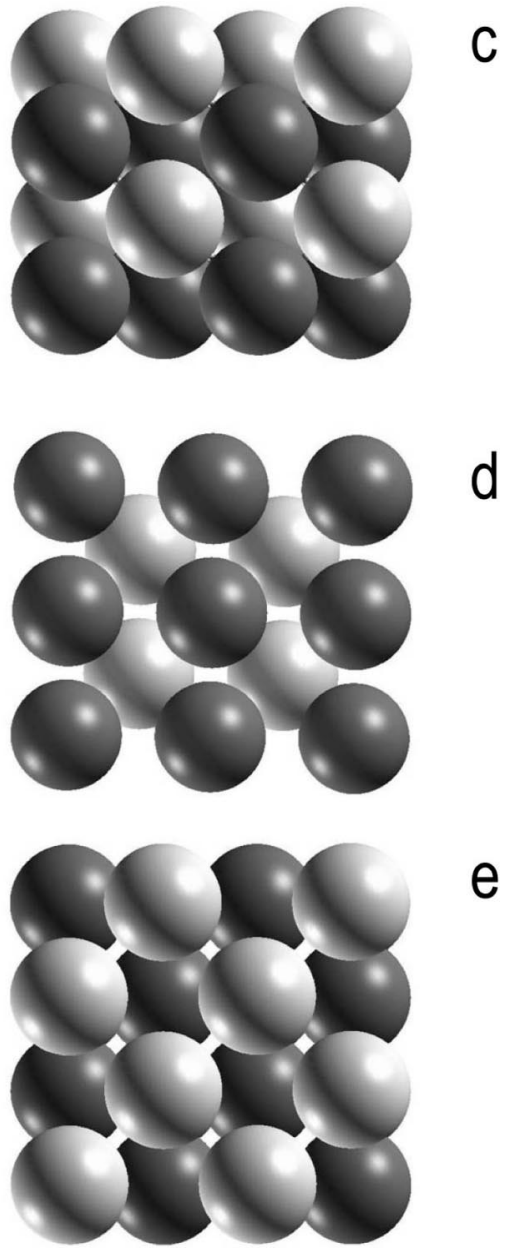

FIG. 1. (a) $\mathrm{CuAu} L 1_{0}$-type tetragonal structure of $\mathrm{PdZn}$ and PtZn alloys. Light spheres: Zn, dark spheres: Pd. Also shown are top views of the surfaces (111) (b), (100) (c), (110) (d), and (001) (e). 
TABLE I. Calculated and experimental parameters $a$ and $c(\AA)$ of a tetragonal or fcc lattice as well as their differences $\Delta a$ and $\Delta c$ $(\%)$. Also shown are calculated cohesive energies $E_{B}$ (eV/atom).

\begin{tabular}{|c|c|c|c|c|c|c|c|}
\hline \multirow[t]{2}{*}{ Material } & \multicolumn{2}{|c|}{$a$} & \multirow[t]{2}{*}{$\Delta a$} & \multicolumn{2}{|c|}{$c$} & \multirow[t]{2}{*}{$\Delta c$} & \multirow[t]{2}{*}{$E_{B}$} \\
\hline & Calc. & Exp. & & Calc. & Exp. & & \\
\hline $\mathrm{Cu}$ & 3.628 & 3.6147 & 0.4 & & & & -3.51 \\
\hline $\mathrm{Pd}$ & 3.954 & 3.8907 & 1.6 & & & & -3.70 \\
\hline $\mathrm{Pt}$ & 3.985 & 3.9239 & 1.6 & & & & -5.57 \\
\hline $\mathrm{Zn}$ & 2.682 & 2.6649 & 0.6 & 4.856 & 4.9468 & -1.8 & -1.10 \\
\hline $\mathrm{PdZn}$ & 4.148 & 4.11 & 0.9 & 3.385 & 3.35 & 1.0 & -2.98 \\
\hline PtZn & 4.087 & 4.03 & 1.4 & 3.517 & 3.47 & 1.4 & -3.91 \\
\hline
\end{tabular}

move inward (into the bulk) while $\mathrm{Zn}^{1}$ atoms relax outward (to the surface) with respect to the computed bulk-terminated geometry. The vertical displacement $\Delta z$ is larger for $\mathrm{Pd}^{1}$ than for $\mathrm{Zn}^{1}$ atoms: the values for the (111) surface are $0.11 \AA$ and $0.07 \AA$, respectively; the corresponding values of the (100) surface are 0.07 and $0.05 \AA$. For the (111) and (100) surfaces, opposite displacements of the atoms cause a notable surface corrugation, $z\left(\mathrm{Zn}^{1}\right)-z\left(\mathrm{Pd}^{1}\right), 0.17$ and $0.12 \AA$, respectively. $\mathrm{Pd}^{2}$ and $\mathrm{Zn}^{2}$ atoms are displaced notably less than $\mathrm{Pd}^{1}$ and $\mathrm{Zn}^{1}$; thus, the layer spacing $d_{12}^{\mathrm{Pd}}$ between $\mathrm{Pd}^{1}$ and $\mathrm{Pd}^{2}$ atoms is reduced by $0.10 \AA$ for the (111) and $0.05 \AA$ for the (100) surface. At variance, relaxation increases the spacing between the outer two $\mathrm{Zn}$ layers by $0.10 \AA$ for the (111) surface and by $0.04 \AA$ for the (100) surface, mainly due to outward displacement of $\mathrm{Zn}^{1}$.

PdZn (001) and (110) surfaces are terminated by either Pd atoms $(001)^{\mathrm{Pd}}$ and $(110)^{\mathrm{Pd}}$ or $\mathrm{Zn}$ atoms $(001)^{\mathrm{Zn}}$ and $(110)^{\mathrm{Zn}}$. The relaxation pattern of the two Pd-terminated surfaces and that of the two Zn-terminated surfaces is quite similar (Table III). Thus, we only discuss the surfaces (110) ${ }^{\mathrm{Pd}}$ and (110) ${ }^{\mathrm{Zn}}$. For $(110)^{\mathrm{Pd}}$, relaxation leads to an inward displacement of both $\mathrm{Pd}^{1}$, by $0.09 \AA$, and $\mathrm{Pd}^{2}$, by $0.01 \AA$, whereas outward movements are calculated for $\mathrm{Zn}^{1}$, by $0.09 \AA$, and $\mathrm{Zn}^{2}$, by $0.03 \AA$. As on the surfaces (111) and (100), the inward displacement of $\mathrm{Pd}^{1}$ is larger than that of $\mathrm{Pd}^{2}$, resulting in a reduction of $d_{12}^{\mathrm{Pd}}$ by $0.08 \AA$; the outward relaxation of $\mathrm{Zn}$ increases $d_{12}^{\mathrm{Zn}}$ by $0.06 \AA$. In the Zn-terminated (110) surface, $\mathrm{Zn}^{1}$ is displaced inward by $0.07 \AA$, which is accompanied by an outward relaxation of $\mathrm{Pd}^{1}$, by $0.06 \AA$, and $\mathrm{Pd}^{2}$, by $0.02 \AA$.

TABLE II. Calculated interlayer distances $d_{\text {int }}$ for various crystal planes and nearest-neighbor distances $d_{M-M}$ and $d_{M-Z n}$ for optimized geometries of Pd, Pt, PdZn, and PtZn (in $\AA$ ).

\begin{tabular}{lcccc}
\hline \hline Parameters & Pd & Pt & PdZn & PtZn \\
\hline$d_{\text {int }}^{001}$ & 1.977 & 1.993 & 1.693 & 1.759 \\
$d_{\text {int }}^{110}$ & 1.398 & 1.409 & 1.467 & 1.455 \\
$d_{\text {int }}^{1100}$ & 1.977 & 1.993 & 2.074 & 2.044 \\
$d_{\text {int }}^{111}$ & 2.283 & 2.301 & 2.217 & 2.233 \\
$d_{M-Z n}{ }^{a}$ & & & 2.677 & 2.696 \\
$d_{M-M}{ }^{\mathrm{b}}$ & 2.796 & 2.818 & 2.933 & 2.890 \\
\hline \hline
\end{tabular}

${ }^{\mathrm{a}} M=\mathrm{Pd}, \mathrm{Pt}$.

${ }^{\mathrm{b}} d_{M-M}=d_{\mathrm{Zn}-\mathrm{Zn}}$.
TABLE III. Vertical atomic displacements $\Delta z$, spacings $d_{i j}^{M}$ between metal centers $M$ of the $i$ th and the $j$ th layers, and relaxation energy $E_{\mathrm{rlx}}$ for various surfaces of PdZn alloy. Distances in $\AA$, energies in meV.

\begin{tabular}{lccccccc}
\hline \hline Parameter & $(111)$ & $(100)$ & $(110)^{\mathrm{Pd}}$ & $(110)^{\mathrm{Zn}}$ & $(001)^{\mathrm{Pd}}$ & $(001)^{\mathrm{Zn}}$ \\
\hline$\Delta z\left(\mathrm{Pd}^{1}\right)^{\mathrm{a}}$ & -0.107 & -0.069 & -0.088 & 0.064 & -0.060 & 0.046 \\
$\Delta z\left(\mathrm{Zn}^{1}\right)$ & 0.066 & 0.047 & 0.086 & -0.074 & 0.076 & -0.028 \\
$\Delta z\left(\mathrm{Pd}^{2}\right)$ & -0.005 & -0.024 & -0.011 & 0.023 & -0.017 & -0.008 \\
$\Delta z\left(\mathrm{Zn}^{2}\right)$ & -0.031 & 0.007 & 0.028 & -0.004 & 0.006 & -0.007 \\
$d_{12}^{\mathrm{Pd}}$ & 2.115 & 2.029 & 2.856 & 2.974 & 3.343 & 3.440 \\
$d_{12}^{\mathrm{Zn}}$ & 2.313 & 2.114 & 2.992 & 2.863 & 3.455 & 3.365 \\
$d_{23}^{\mathrm{Pd}}$ & 2.211 & 2.050 & 2.922 & 2.956 & 3.368 & 3.377 \\
$d_{23}^{\mathrm{Zn}}$ & 2.186 & 2.081 & 2.961 & 2.929 & 3.391 & 3.378 \\
$d_{34}^{\mathrm{Pd} b}$ & 2.217 & 2.074 & 2.933 & 2.933 & 3.385 & 3.385 \\
$E_{\mathrm{rlx}}$ & 33 & 16 & 64 & 39 & 51 & 12 \\
\hline \hline
\end{tabular}

${ }^{\mathrm{a}} \mathrm{Pd}^{1}\left(\mathrm{Zn}^{1}\right)$ and $\mathrm{Pd}^{2}\left(\mathrm{Zn}^{2}\right)$ denote $\mathrm{Pd}(\mathrm{Zn})$ atoms of the first and second layers, respectively. $\Delta z$ refers to the atomic displacement perpendicular to the surface. $(110)^{\mathrm{Pd}}\left[(001)^{\mathrm{Pd}}\right]$ and $(110)^{\mathrm{Zn}}$ $\left[(001)^{\mathrm{Zn}}\right]$ represent slabs terminated with $\mathrm{Pd}$ and $\mathrm{Zn}$, respectively. Positive $\Delta z$ values denote an outward displacement, toward the vacuum, negative $\Delta z$ values denote an atom movement in the direction of the bulk.

${ }^{\mathrm{b}} d_{34}^{\mathrm{Pd}}=d_{34}^{\mathrm{Zn}}$.

The interlayer spacings $d_{12}^{\mathrm{Pd}}$ and $d_{23}^{\mathrm{Pd}}$ are enlarged by 0.04 and $0.02 \AA$, whereas $d_{12}^{\mathrm{Zn}}$ shrinks by $0.07 \AA$ and $d_{23}^{\mathrm{Zn}}$ remains unchanged.

The data in Table III show that $\mathrm{Zn}$ atoms shift upward and $\mathrm{Pd}$ atoms move downward on the stoichiometric (111) and (100) surfaces. This can be rationalized with the smaller surface energy of $\mathrm{Zn}$ (see next subsection). The slab thickness expands because of the outward displacement of $\mathrm{Zn}^{1}$. When the top layer is formed by only atoms of one element, as on the surfaces (110) and (001), the surface atoms relax inward and the slab becomes thinner.

Relaxation of the top layer contributes the most to the relaxation energy. Therefore, larger displacements of $\mathrm{Pd}^{1}$ or $\mathrm{Zn}^{1}$ atoms correspond to larger relaxation energies. For instance, the changes in the interlayer distances $d_{12}^{\mathrm{Pd}}$ and $d_{12}^{\mathrm{Zn}}$ of the (111) surface, $0.10 \AA$, are about twice as large as those of the (100) surface; the corresponding relaxation energies, though rather small, are 33 and $16 \mathrm{meV}$ (Table III). The finding that the Pd-terminated surfaces (110) and (001) relax somewhat more than Zn-terminated surfaces is in line with the larger surface energy of $\mathrm{Pd}$ compared to $\mathrm{Zn}$.

\section{Relative stability of the alloy surfaces}

In Table IV, we present calculated surface energies for various surfaces of $\mathrm{PdZn}$ and PtZn alloys and for pure metals together with pertinent experimental data. ${ }^{37}$ For the pure metals considered, the calculated surface energies are notably lower than the experimental values. For instance, the computed $\mathrm{Pd}(111)$ surface energy $1.38 \mathrm{~J} \mathrm{~m}^{-2}$ is only about two-thirds of the experimental value $2.05 \mathrm{~J} \mathrm{~m}^{-2} \cdot{ }^{37}$ At the experimental geometry, the difference between calculated 
TABLE IV. Calculated characteristics of pertinent surfaces of various materials: surface area $S$ of a unit cell and surface energy $\gamma$. Experimental surface energies are listed where available.

\begin{tabular}{|c|c|c|c|c|c|}
\hline Material & Surface & $S\left(\AA^{2}\right)$ & $\gamma\left(\mathrm{eV} /\right.$ atom $\left.^{-1}\right)$ & $\gamma\left(\mathrm{J} \mathrm{m}^{-2}\right)$ & $\gamma_{\exp }\left(\mathrm{J} \mathrm{m}^{-2}\right)$ \\
\hline $\mathrm{Pd}^{\mathrm{b}}$ & (111) & 13.11 & 0.53 & 1.30 & \\
\hline $\mathrm{Pd}^{\mathrm{c}}$ & (111) & 13.11 & 0.79 & 1.92 & \\
\hline $\mathrm{Pd}$ & (110) & 11.06 & 1.11 & 1.61 & \\
\hline $\mathrm{Pd}$ & (100) & 15.63 & 0.75 & 1.53 & \\
\hline $\mathrm{Pd}$ & (111) & 13.56 & 0.59 & 1.38 & 2.05 \\
\hline $\mathrm{Pt}$ & (111) & 13.76 & 0.64 & 1.50 & 2.48 \\
\hline $\mathrm{Cu}$ & (110) & 9.31 & 0.92 & 1.58 & \\
\hline $\mathrm{Cu}$ & (100) & 13.16 & 0.63 & 1.53 & \\
\hline $\mathrm{Cu}$ & (111) & 11.40 & 0.48 & 1.34 & 1.83 \\
\hline $\mathrm{Zn}$ & (0001) & 6.23 & 0.14 & 0.35 & 0.99 \\
\hline $\mathrm{PdZn}$ & (111) & 13.14 & 0.48 & 1.17 & \\
\hline$P d Z n^{d}$ & (111) & 13.14 & 0.46 & 1.11 & \\
\hline $\mathrm{PdZn}$ & (100) & 14.04 & 0.54 & 1.23 & \\
\hline $\mathrm{PdZn}$ & (001) & 8.60 & 0.89 & 1.65 & \\
\hline $\mathrm{PdZn}$ & (110) & 9.93 & 0.98 & 1.57 & \\
\hline PtZn & (111) & 13.25 & 0.55 & 1.34 & \\
\hline PtZn & (100) & 14.38 & 0.70 & 1.56 & \\
\hline PtZn & $(001)$ & 8.35 & 0.92 & 1.76 & \\
\hline PtZn & (110) & 10.17 & 1.15 & 1.82 & \\
\hline
\end{tabular}

${ }^{\mathrm{a}}$ Reference 38 .

${ }^{\mathrm{b}} \mathrm{GGA}$ result at experimental geometry.

${ }^{c}$ LDA result at experimental geometry.

${ }^{\mathrm{d}}$ Based on one-sided relaxation of a four-layer slab; Eq. (2) applied in modified form.

$1.30 \mathrm{~J} \mathrm{~m}^{-2}$ and experimental values is even larger. For the same geometry, using a denser $(11 \times 11 \times 1)$ mesh and the tetrahedron method with Blöchl corrections for partial occupancies, ${ }^{28}$ one obtains essentially the same value $1.30 \mathrm{~J} \mathrm{~m}^{-2}$. As already mentioned (Sec. II B), relaxation slightly reduces (by 4\%, Table IV) the calculated surface energy of $\operatorname{PdZn}(111)$, in line with results for other metal systems. ${ }^{33-35}$ GGA is well known to underestimate surface energies. ${ }^{38}$ With the local density approximation (LDA), ${ }^{39}$ we determined the surface energy of $\operatorname{Pd}(111)$ to $1.92 \mathrm{~J} \mathrm{~m}^{-2}$, which is $50 \%$ larger than the GGA result and close to the experimental value. Thus, the calculated PW91 surface energies for PdZn alloy likely are also notably underestimated. Nevertheless, the relative magnitude of surface energies is expected to be predicted correctly. For example, the ordering of the calculated surface energies of the (111) surfaces of $\mathrm{Cu}$, $\mathrm{Pd}$, and Pt agrees with experiment (Table IV). Furthermore, our predicted stability ordering of (111), (100), and (110) surfaces for $\mathrm{Cu}$ and $\mathrm{Pd}$ is the same as in other calculations. ${ }^{14}$

Now, we compare the surface tension of four surfaces of 1:1 PdZn alloy. According to the "bond-cutting" model, ${ }^{40}$ the surface energy anisotropy is proportional to the ratio of broken bonds at the surfaces under comparison. For materials of fcc structure, three bonds of each atom at the (111) surface are broken, four bonds at the (001) surface, and six bonds at the (110) surface. Thus, ratios $\gamma_{001} / \gamma_{111}$ $\left(=\gamma_{100} / \gamma_{111}\right)$ and $\gamma_{110} / \gamma_{111}$ of surface energies are pre- dicted at 1.3 and 2.0, respectively. For metallic $\mathrm{Pd}$ and $\mathrm{Cu}$, our calculated surface energy anisotropy is in line with the bond-cutting model $\gamma_{001} / \gamma_{111}=1.3$ and $\gamma_{110} / \gamma_{111}=1.9$ (Table IV). For PdZn, we derived the following ratios of surface energies: $\gamma_{100} / \gamma_{111}=1.1, \quad \gamma_{001} / \gamma_{111}=1.9$, and $\gamma_{110} / \gamma_{111}=2.0$ (Table IV). Compared with Pd metal, the ratio $\gamma_{001} / \gamma_{111}$ of $\mathrm{PdZn}$ is about $45 \%$ larger. On the other hand, the surface energy of the (100) surface of $\mathrm{PdZn}$ is only slightly larger than that of (111) surface; thus, $\gamma_{100} / \gamma_{111}$ of $\mathrm{PdZn}$ is $15 \%$ smaller than in the case of metallic Pd.

As mentioned before, each type of atoms in $\mathrm{PdZn}$ is surrounded by eight nearest neighbors of the other atom type; four homonuclear bonds, about $10 \%$ longer, extend to the next-nearest neighbors. If both types of bonds $A-A$ and $A-B$ would contribute equally to the surface energy, then one has three broken bonds at (111) surface, four at the (001) and (100) surfaces and six at the (110) surface, just as in materials with fcc structure. These values would translate into the ratios $\gamma_{001} / \gamma_{100} \sim 1$ and $\gamma_{001} / \gamma_{111} \sim 1.3$, in disagreement with our calculated values $\gamma_{001} / \gamma_{100}=1.6$ and $\gamma_{001} / \gamma_{111}$ $=1.9$. On the other hand, if the surface energy of $\mathrm{PdZn}$ alloy is predominantly determined by the shorter (and presumably stronger) $\mathrm{Pd}-\mathrm{Zn}$ bonds, then the bond-cutting model yields equal ratios $\gamma_{001} / \gamma_{100}=\gamma_{001} / \gamma_{111}=2$, close to the explicitly calculated ratios of 1.6 and 1.9. [The number of broken Pd-Zn bonds per atom at the (111), (100), (001), and (110) surfaces is 2, 2, 4 and 4, respectively.] Thus, the model of stronger $\mathrm{Pd}-\mathrm{Zn}$ bonds allows a rationalization of the surface energy anisotropy calculated for PdZn. Our calculated surface tension values for PtZn (Table IV) do not follow the prediction of the bond-cutting model quite as closely as for PdZn: $\gamma_{001} / \gamma_{100}=1.3$, and $\gamma_{100} / \gamma_{111}=2.1$. This finding is likely due to the reduced difference in nearest-neighbor $(A-B)$ and next-nearest-neighbor $(A-A)$ distances compared to $\mathrm{PdZn}$ (see Sec. III A).

The results of Table IV show that the surface energy of the alloys considered lies between the surface energies of their components, but is not their composition-weighted average. In other words, there does not seem to hold a linear relationship between the surface energies of an alloy and its components. For example, the surface energy values of $\mathrm{Pd}(111)$ and $\mathrm{Zn}(0001)$ are 0.59 and $0.14 \mathrm{eV} /$ atom, respectively. The value of $\operatorname{PdZn}(111)$ is $0.48 \mathrm{eV} /$ atom, notably larger than the corresponding average $0.36 \mathrm{eV} /$ atom. From another point of view we note that the surface energy of $\mathrm{Pt}(111)$ is computed by $8 \%$ larger than that of $\mathrm{Pd}(111)$ whereas the surface energy of $\mathrm{PtZn}(111)$ is $13 \%$ larger than the value of $\mathrm{PdZn}(111)$ (Table IV).

$\mathrm{PdZn}$ and PtZn alloys are expected to exhibit certain analogies in the electronic structure and chemisorption properties to metallic $\mathrm{Cu}$. Thus, we compare the surface energies of $\mathrm{Cu}$ with that of $\mathrm{PdZn}$ and PtZn alloys. Based on the larger (by absolute value) bulk energy of PtZn than of PdZn (Table I), Pt-Zn bonds should be stronger than $\mathrm{Pd}-\mathrm{Zn}$ bonds. Therefore, from the bond-cutting model ${ }^{40}$ one expects the energy of a PtZn surface to be larger than the energy of the corresponding $\mathrm{PdZn}$ surface. Our calculated surface energies comply with this assumption: $\gamma_{\mathrm{PtZn}(111)} / \gamma_{\mathrm{PdZn}(111)}$ $=1.15$ and $\gamma_{\operatorname{PtZn}(100)} / \gamma_{\operatorname{PdZn}(100)}=1.30$. The surface energies 
TABLE V. Calculated energies $\varepsilon_{\mathrm{BC}}$ (in $\mathrm{eV}$ ) of the centers of the $\mathrm{Zn}$ valence $s$ and $d$ bands in various solids and films.

\begin{tabular}{lcc}
\hline \hline & \multicolumn{3}{c}{$\varepsilon_{\mathrm{BC}}$} \\
& $s$ & $d$ \\
\hline Zn (bulk) & -1.05 & -6.65 \\
PdZn (bulk) & -1.90 & -6.66 \\
PtZn (bulk) & -2.07 & -6.54 \\
PdZn (100) & -2.33 & -6.63 \\
PdZn (111) & -2.16 & -6.49 \\
PtZn (100) & -2.32 & -6.44 \\
PtZn (111) & -2.00 & -6.35 \\
\hline \hline
\end{tabular}

of $\mathrm{PdZn}(111)$ and $\mathrm{Cu}(111)$ are equal, $0.48 \mathrm{eV} /$ atom, and for the (110) surfaces they are rather similar, $0.92 \mathrm{eV} /$ atom for $\mathrm{Cu}(110)$ and $0.98 \mathrm{eV} /$ atom for $\mathrm{PdZn}(110)$. Calculations reveal that the (111) surfaces of $\mathrm{PdZn}$ and $\mathrm{PtZn}$ are the most stable ones (Table IV); these surfaces will be exposed favorably at thermodynamic equilibrium. From the close surface energy values of $\mathrm{PdZn}(100)$ and $\mathrm{PdZn}(111)$ one expects that both types of surfaces are exposed and thus more likely to be observed. In fact, XRD peaks of $\mathrm{PdZn}(111)$ and $\mathrm{PdZn}(100)$ provide indirect support for this conclusion. ${ }^{41}$

Finally, we note in passing that segregation of $\mathrm{Zn}$ on the surfaces of $\mathrm{PdZn}$ alloy has been postulated, ${ }^{10}$ based on the notably larger surface energy of Pd compared to $\mathrm{Zn}$. As we will demonstrate elsewhere, ${ }^{42}$ segregation of $\mathrm{Zn}$ on the $\mathrm{PdZn}$ alloy is not energetically favorable due to the large energy required to break $\mathrm{Pd}-\mathrm{Zn}$ bonds.

\section{Electronic structure}

Finally, we discuss the electronic structure of the various surfaces and bulk of the materials under study. Surface DOS were calculated for the first-layer atoms of the unrelaxed seven-layer slabs; the electronic structure of the second-layer (subsurface) atoms is already very similar to that of the corresponding bulk. Band energies will be given relative to the Fermi energies.

Table V summarizes the energies $\epsilon_{\mathrm{BC}}$ of the band centers (BC's) of Zn. Alloying notably shifts the center of $\mathrm{Zn} 4 s$ band from $-1.05 \mathrm{eV}$ in metallic $\mathrm{Zn}$ to -1.90 and $-2.07 \mathrm{eV}$ in bulk PdZn and PtZn, respectively. On the other hand, $\varepsilon_{\mathrm{BC}}$ values for $\mathrm{Zn} 3 d$ are predicted for the alloys to be close to those of bulk $\mathrm{Zn}$. Thus, not unexpectedly, $\mathrm{Zn} 4 s$ electrons play a central role in alloy formation. Photoemission spectra show that in $\mathrm{Pd}_{9.3} \mathrm{Zn}_{0.9}$ the binding energy of $\mathrm{Zn}$ $3 d$ bands is lowered by $0.65 \mathrm{eV}$ (Ref. 10) whereas in $\mathrm{Pd}_{11.8} \mathrm{Zn}_{8.6}$ alloy film no significant shift of the $\mathrm{Zn} 3 d$ peak relative to that of metal $\mathrm{Zn}$ was found, in line with our calculated data. Going from bulk PdZn to its (111) and (100) surfaces, $4 s$-BC's of Zn tend to shift down while $3 d$-BC's shift up.

As shown in Table VI, alloying increases the energy of the $\mathrm{Pd} 5 s$ and $\mathrm{Pt} 6 s$ BC's by $0.5-0.6 \mathrm{eV}$ relative to the position in bulk $\mathrm{Pd}(-2.02 \mathrm{eV})$ and $\mathrm{Pt}(-2.52 \mathrm{eV})$. This renders the two $s$-BC values closer to $\mathrm{Cu} 4 s$-BC energy $(-1.45 \mathrm{eV})$. Calculations on $\mathrm{PdV}$ and $\mathrm{PdRe}$ alloys revealed a lowering of
TABLE VI. Calculated energies $\varepsilon_{\mathrm{BC}}$ (in $\mathrm{eV}$ ) of the centers of the metal $(\mathrm{Cu}, \mathrm{Pd}, \mathrm{Pt})$ valence $s$ and $d$ bands in various solids and films. Also shown are the local densities of states $N_{F}$ at the Fermi energy (states/eV).

\begin{tabular}{lcccc}
\hline \hline & \multicolumn{2}{c}{$\varepsilon_{\mathrm{BC}}$} & \multicolumn{2}{c}{$N_{F}$} \\
& $s$ & $d$ & $s$ & $d$ \\
\hline Pd (bulk) & -2.02 & -1.81 & 0.02 & 2.43 \\
Pt (bulk) & -2.52 & -2.45 & 0.02 & 2.16 \\
Cu (bulk) & -1.45 & -2.44 & 0.04 & 0.13 \\
PdZn (bulk) & -1.46 & -2.51 & 0.03 & 0.15 \\
PtZn (bulk) & -2.01 & -2.45 & 0.03 & 0.22 \\
Pd (111) & -1.38 & -1.54 & 0.03 & 2.03 \\
Pt (111) & -2.68 & -2.02 & 0.03 & 1.81 \\
Cu (100) & -1.26 & -2.13 & 0.05 & 0.10 \\
Cu (111) & -1.35 & -2.22 & 0.05 & 0.12 \\
PdZn (100) & -0.93 & -2.17 & 0.26 & 0.40 \\
PdZn (111) & -1.10 & -2.04 & 0.08 & 0.21 \\
PtZn (100) & -1.73 & -2.00 & 0.07 & 0.21 \\
PtZn (111) & -1.82 & -1.88 & 0.05 & 0.30 \\
\hline \hline
\end{tabular}

the Pd $4 d$-BC energy due to alloying. ${ }^{43,44}$ We calculated a lowering of $0.70 \mathrm{eV}$ for $\mathrm{Pd} 4 d$-BC energy in $\mathrm{PdZn}$ alloy relative to bulk Pd. Thus, both $4 d$ and $5 s$ states of Pd contribute significantly to the formation of intermetallic bonds by alloying with $\mathrm{Zn}$. Note that the bulk valence $d$-BC energies of $\mathrm{Cu}$ and $\mathrm{PdZn}$ and $\mathrm{PtZn}$ alloys are very similar: $-2.44,-2.51$, and $-2.45 \mathrm{eV}$, respectively. Due to the reduction of the coordination number of atoms on the alloy surfaces, BC's energies of all bands are increased relative to the corresponding bulk. For example, for $\mathrm{PdZn}$ this shift is $0.3-0.6 \mathrm{eV}$ (Table VI).

Valence $d$ states of transition metal atoms in the systems under study are of particular importance for the surface reactivity, which is the ultimate target of our investigations of alloy catalysts. Thus, these states deserve closer inspection. The valence $d$-band widths at half maximum of bulk Pd, Pt, PdZn, and PtZn are 4.5, 4.8, 2.0, and $2.8 \mathrm{eV}$, respectively. The $d$-band width is inversely proportional to the third to fifth power of the distance between the atoms. ${ }^{45,46}$ As the nearest-neighbor $M-M$ distance in the $M \mathrm{Zn}(M=\mathrm{Pd}, \mathrm{Pt})$ alloys is longer than in bulk M (see Sec. III A and Table II), one expects a reduction of the valence $M d$-band width in $M Z n$ alloys, in line with our calculated data. The local density of states $N_{F}$ at the Fermi level for bulk Pd and Pt is 2.43 and 2.16 states/eV, respectively (Table VI). Due to the narrower $d$-band width, these values dramatically decrease to $0.15(\mathrm{PdZn})$ and $0.22(\mathrm{PtZn})$ states/eV, very close to 0.13 states/eV for $\mathrm{Cu}$. Comparing the local $M d$-DOS profiles of $\mathrm{PdZn}$ and PtZn, we note that they are more similar to the profile of $\mathrm{Cu}$ than to those of pure Pd and Pt metals (which are similar to each other, Fig. 2). The latter two observations concerning the close similarity in the electronic structure of the alloys and $\mathrm{Cu}$ are crucial for rationalizing why these different materials exhibit similar surface reactivity. On the other hand, this result is in line with very simple arguments: if one mixes $d^{10}$ atoms ( $\mathrm{Pd}$ or $\mathrm{Pt}$ ) with $d^{10} s^{2}$ atoms ( $\left.\mathrm{Zn}\right)$ one 

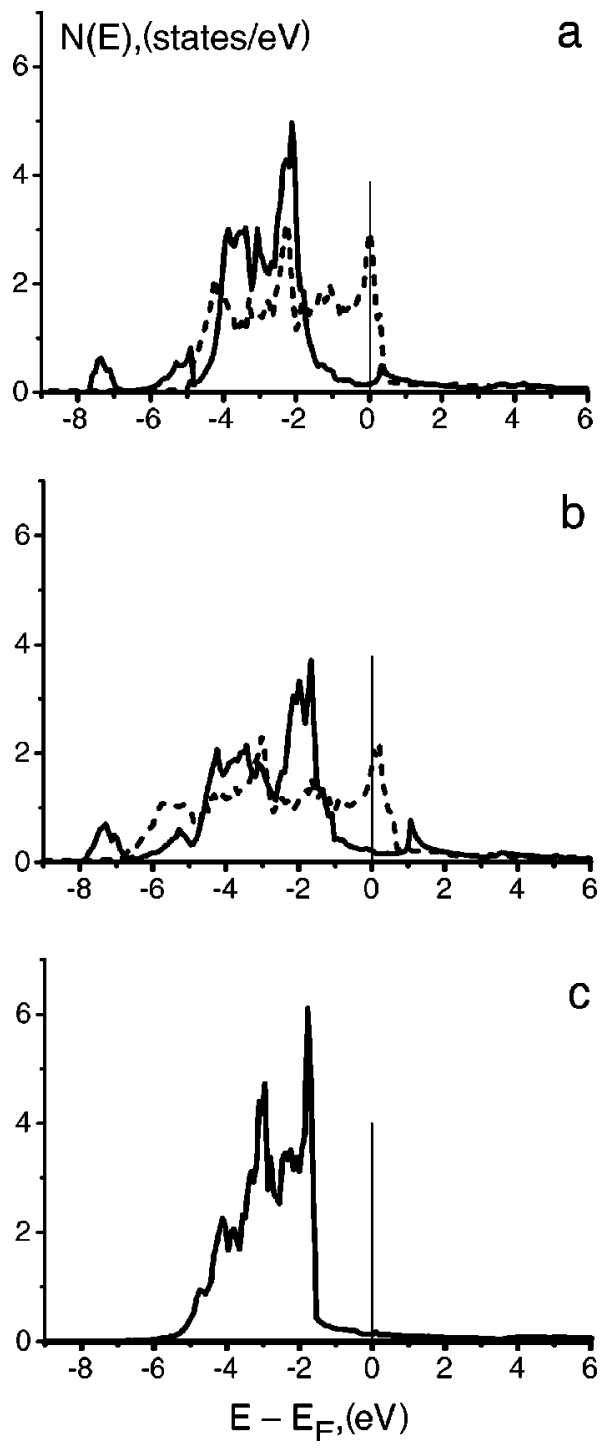

FIG. 2. Local density of states of pertinent metals and alloys: (a) Pd $4 d$ band of bulk Pd (dashed) and PdZn (solid); (b) Pt $5 d$-band of bulk Pt (dashed) and PtZn (solid); (c) $\mathrm{Cu} 3 d$-band of bulk $\mathrm{Cu}$.

obtains an alloy with electron configuration of the "average" atom $d^{10} s^{1}$, which is the electron configuration of $\mathrm{Cu}$ atoms.

The abovementioned trends in the surface reactivity can be illustrated with the adsorption strength of probe $\mathrm{CO}$ molecules. According to our periodic slab PW91 calculations for a CO coverage $1 / 4,{ }^{47}$ the most stable surface $\mathrm{PdZn}(111)$ binds single $\mathrm{CO}$ molecules much weaker, $1.0 \mathrm{eV}$, than $\mathrm{Pd}(111), 1.9 \mathrm{eV}$; the former value is close to the CO adsorption energy computed on the $\mathrm{Cu}(111)$ surface, $0.9 \mathrm{eV}$. These adsorption energy values are in agreement with the experimental result that the maximum desorption temperature of $\mathrm{CO}$ on $\mathrm{Pd}_{5.8} \mathrm{Zn}_{10.4}$ film is $\sim 220 \mathrm{~K}$ whereas on pure $\mathrm{Pd}(111)$ a maximum temperature of $\sim 460 \mathrm{~K}$ is observed, implying a decrease of $0.7 \mathrm{eV}$ in the adsorption energy. ${ }^{10}$

\section{CONCLUSIONS}

We carried out a density functional study on the 1:1 alloys $\mathrm{PdZn}$ and PtZn as well as on the metals $\mathrm{Pd}, \mathrm{Pt}$, and $\mathrm{Cu}$, applying a band structure method to bulk materials and slab models. We optimized the lattice parameters of the alloys assuming the experimentally found structure of $\mathrm{CuAu}-L 1_{0}$. From the calculated cohesive energies it follows that $\mathrm{PdZn}$ and PtZn alloy formation is exothermic; both processes are accompanied by an essentially equal energy gain. The (111) surfaces of PdZn and PtZn alloys were calculated to have the smallest surface energies; the energies of (100) surfaces are only slightly larger. Therefore, (111) surfaces and likely also (100) surfaces will play an important role in the surface chemistry of PdZn and PtZn alloys. The anisotropy of PdZn surface energy correlates well with the number of $\mathrm{Pd}-\mathrm{Zn}$ bonds broken through the surface formation.

To unravel similarities in the performance of the $\mathrm{PdZn}$ and $\mathrm{Cu}$ catalysts we compared characteristic features of the electronic structure of the most stable surfaces $\mathrm{PdZn}(111)$ and $\mathrm{Cu}(111)$, that exhibit essentially equally low calculated surface energies. The local valence $d$-DOS profile of $\mathrm{Pd}$ in $\mathrm{PdZn}$ is found to resemble that of metal $\mathrm{Cu}$ (Fig. 2). Furthermore, the $\mathrm{PdZn}$ alloy formation is accompanied by a significantly reduced width, by $2.5 \mathrm{eV}$, of the valence $d$ band as measured by the full width at half maximum. Also, $\mathrm{Pd}$ in $\mathrm{PdZn}$ exhibits a notably lower density of states at the Fermi level, by 2.28 states/eV, compared to elemental Pd. The resulting parameters, band width $=2.0 \mathrm{eV}$ and the local DOS at the Fermi energy $=0.15$ states/eV, are quite close to the corresponding values of $\mathrm{Cu}, 1.6 \mathrm{eV}$ and 0.13 states/eV, respectively. These findings provide a key for rationalizing the similar surface reactivity of $\mathrm{PdZn}$ and $\mathrm{Cu}$. Analogous arguments are applicable to PtZn alloy materials, which we predict to manifest an electronic structure very much reminiscent of that for PdZn alloy.

\section{ACKNOWLEDGMENTS}

Zhao-Xu Chen gratefully acknowledges a fellowship of the Alexander von Humboldt foundation. This work was supported by Deutsche Forschungsgemeinschaft and Fonds der Chemischen Industrie.
*Corresponding author.

${ }^{1}$ J.M. Ogden, Annu. Rev. Energy Environ. 24, 227 (1999).

${ }^{2}$ D.L. Trimm and Z.I. Önsan, Catal. Rev. 43, 31 (2001).

${ }^{3}$ N. Takezawa and N. Iwasa, Catal. Today 36, 45 (1997).

${ }^{4}$ M.L. Cubeiro and J.L.G. Fierro, J. Catal. 179, 150 (1998).

${ }^{5}$ N. Iwasa, N. Ogawa, S. Masuda, and N. Takezawa, Bull. Chem. Soc. Jpn. 71, 1451 (1998).
${ }^{6}$ N. Iwasa, T. Mayanagi, S. Masuda, and N. Takezawa, React. Kinet. Catal. Lett. 69, 355 (2000).

${ }^{7}$ N. Iwasa, T. Mayanagi, N. Ogawa, K. Sakata, and N. Takezawa, Catal. Lett. 54, 199 (1998).

${ }^{8}$ J.A. Rodriguez, Surf. Sci. Rep. 24, 223 (1996).

${ }^{9}$ B.E. Nieuwenhuys, Surf. Rev. Lett. 3, 1869 (1996), and references therein. 
${ }^{10}$ J.A. Rodriguez, J. Phys. Chem. 98, 5758 (1994).

${ }^{11}$ J.A. Rodriguez and M. Kuhn, J. Phys. Chem. 100, 381 (1996).

${ }^{12}$ U. Birkenheuer, J.C. Boettger, and N. Rösch, Surf. Sci. 341, 103 (1995).

${ }^{13}$ J.C. Boettger, J.R. Smith, U. Birkenheuer, N. Rösch, S.B. Trickey, J.R. Sabin, and S.P. Apell, J. Phys.: Condens. Matter 10, 893 (1998), and references therein.

${ }^{14}$ L. Vitos, A.V. Ruban, H.L. Skriver, and J. Kollár, Surf. Sci. 411, 186 (1998), and references therein.

${ }^{15}$ S.Y. Choi, Y.S. Kwon, T.H. Rho, and S.C. Hong, J. Korean Phys. Soc. 37, 104 (2000).

${ }^{16}$ V.M. Kuznetsov, R.I. Kadyrov, and G.E. Rudenskii, J. Mater. Sci. Technol. 14, 320 (1998).

${ }^{17}$ G. Kresse and J. Furthmüller, Phys. Rev. B 54, 11169 (1996).

${ }^{18}$ G. Kresse and J. Hafner, Phys. Rev. B 48, 13115 (1993).

${ }^{19}$ G. Kresse and J. Hafner, Phys. Rev. B 49, 14251 (1994).

${ }^{20}$ G. Kresse and J. Hafner, Phys. Rev. B 47, 558 (1993).

${ }^{21}$ G. Kresse and J. Furthmüller, Comput. Mater. Sci. 6, 15 (1996).

${ }^{22}$ J.P. Perdew, J.A. Chevary, S.H. Vosko, K.A. Jackson, M.R. Pederson, D.J. Singh, and C. Fiolhais, Phys. Rev. B 46, 6671 (1992).

${ }^{23}$ P.E. Blöchl, Phys. Rev. B 50, 17953 (1994).

${ }^{24}$ G. Kresse and D. Joubert, Phys. Rev. B 59, 1758 (1999).

${ }^{25}$ H.J. Monkhorst and J.D. Pack, Phys. Rev. B 13, 5188 (1976).

${ }^{26}$ M. Methfessel and A.T. Paxton, Phys. Rev. B 40, 3616 (1989).

${ }^{27}$ M. Hansen, Constitution of Binary Alloys, 2nd ed. (McGraw Hill, New York, 1958).

${ }^{28}$ P.E. Blöchl, O. Jepsen, and O.K. Andersen, Phys. Rev. B 49, 16223 (1994).

${ }^{29}$ Periodensystem der Elemente (VCH, Weinheim, 1989).
${ }^{30}$ J.C. Boettger, Phys. Rev. B 49, 16798 (1994).

${ }^{31}$ J.G. Gay, J.R. Smith, R. Richter, F.J. Arlinghaus, and R.H. Wagoner, J. Vac. Sci. Technol. A 2, 931 (1983).

${ }^{32}$ V. Fiorentini and M. Methfessel, J. Phys.: Condens. Matter 8, 6525 (1996).

${ }^{33}$ P.J. Feibelman and D.R. Hamann, Surf. Sci. 234, 377 (1990).

${ }^{34}$ M. Mansfield and R.J. Needs, Phys. Rev. B 43, 8829 (1991).

${ }^{35}$ P.J. Feibelman, Phys. Rev. B 46, 2532 (1992).

${ }^{36} \mathrm{~K}$. Lonsdale, International Tables for X-Ray Crystallography (Kynoch, Birmingham, 1962), Vol. 3.

${ }^{37}$ F. R. de Boer, R. Boom, W. C.M. Mattens, A. R. Miedema, and A. K. Niessen, Cohesion in Metals (North-Holland, Amsterdam, 1988).

${ }^{38}$ S. Kurth, J.P. Perdew, and P. Blaha, Int. J. Quantum Chem. 75, 889 (1999), and references therein.

${ }^{39}$ J.P. Perdew and A. Zunger, Phys. Rev. B 23, 5048 (1981).

${ }^{40}$ D. McLachlan, Acta Metall. 5, 111 (1975).

${ }^{41}$ M-H. Park, L.C. Wang, and C.J. Palmstrøm, J. Appl. Phys. 81, 2720 (1997).

${ }^{42}$ Z.-X. Chen, K. M. Neyman, and N. Rösch (unpublished).

${ }^{43}$ R. Hirschl, J. Hafner, and Y. Jeanvoine, J. Phys.: Condens. Matter 13, 3545 (2001).

${ }^{44}$ V. Pallasana, M. Neurock, L.B. Hansen, and J.K. Nørskov, J. Chem. Phys. 112, 5435 (2000).

${ }^{45}$ O.K. Anderson, W. Klose, and H. Nohl, Phys. Rev. B 17, 1209 (1978).

${ }^{46}$ L. H. Bennett, Theory of Alloy Phase Formation (AIME, Warrendale, PA, 1980), p. 425.

${ }^{47}$ Z.-X. Chen, K. M. Neyman, and N. Rösch (unpublished). 